\title{
Interactive Online Configurator via Boolean Satisfiability Modeling
}

\author{
Tao Tao \\ ttao@cs.unc.edu \\ University of North Carolina at Chapel Hill \\ Chapel Hill, North Carolina, USA
}

\author{
David Plaisted \\ plaisted@cs.unc.edu \\ University of North Carolina at Chapel Hill \\ Chapel Hill, North Carolina, USA
}

\begin{abstract}
Manufacturing companies widely use interactive configurators to specify product variants. These variants are created from different combinations of individual components. Constraints are usually imposed as relations between components to rule out invalid product configurations. The configurator and its underlying algorithm ensure the product specified by the user satisfies all constraints. We provide a method that models the online configurator as a series of highly parallelizable boolean satisfiability problems (SAT). Our methodology is facilitated by state-of-the-art tools such as the Microsoft Z3 theorem prover. Additionally, we confirm that the configurator problem is NP-complete. Hence, using SAT solving is not only natural but canonical.
\end{abstract}

\section{CCS CONCEPTS}

- Software and its engineering $\rightarrow$ Software prototyping; Software prototyping; • Theory of computation $\rightarrow$ Constraint and logic programming.

\section{KEYWORDS}

configurator, satisfiability, parallel computing, user experience, Z3

\section{ACM Reference Format:}

Tao Tao and David Plaisted. 2021. Interactive Online Configurator via Boolean Satisfiability Modeling. In The 12th International Conference on Advances in Information Technology (IAIT2021), fune 29-fuly 1, 2021, Bangkok, Thailand. ACM, New York, NY, USA, 8 pages. https://doi.org/10.1145/3468784. 3468785

\section{INTRODUCTION}

Configurators are widely used by companies such as automotive and personal computer (PC) manufacturers to customize product variants[7][9]. Users can pick different individual choices of components that best suit their needs. For example, a laptop buyer can choose among $4 \mathrm{~GB}, 8 \mathrm{~GB}$, or $16 \mathrm{~GB}$ for the memory component. The number of all possible configurations is $\Pi\left|C_{i}\right|$, where $\left|C_{i}\right|$ is the number of choices for component $C_{i}$. This number is usually very large for industrial-scale applications.

However, not all configurations are valid. For example, in a PC configurator, a particular CPU chip is only compatible with a subset

Permission to make digital or hard copies of part or all of this work for personal or classroom use is granted without fee provided that copies are not made or distributed for profit or commercial advantage and that copies bear this notice and the full citation on the first page. Copyrights for third-party components of this work must be honored.

For all other uses, contact the owner/author(s).

IAIT2021, fune 29-fuly 1, 2021, Bangkok, Thailand

(C) 2021 Copyright held by the owner/author(s).

ACM ISBN 978-1-4503-9012-5/21/06.

https://doi.org/10.1145/3468784.3468785 of motherboards instead of all possible choices of motherboards. A configuration may also be invalid for other higher-order business reasons such as localization or legal restrictions. These business requirements are constraints represented by sets of relations between different components. Specifically, suppose $R: C_{1} \times C_{2}$ is a set of binary constraints in the system. $R$ stipulates that only the relations specified in the set $R$ are permitted between components $C_{1}$ and $C_{2}$. If a user specifies a configuration that contains $\left(c_{1}, c_{2}\right) \notin R$, this violation should be detected, and the entire configuration is invalidated. Constraints range from binary to $k$-ary, where $k$ is the maximum arity of a constraint in the system.

This paper provides a method for creating a feature-rich online configurator based on SAT solving. Special encoding is used in the SAT formula to optimize away time-consuming workflow in traditional algorithms. The user experience is made asynchronous to take full advantage of parallel processing. We built a prototype system based on this idea at a global PC manufacturer. Empirical data show that our method is superior in simplicity and correctness compared to all existing in-house, non-satisfiability approaches.

Our major contributions in this paper are

- Show that encoding a problem as SAT formulas enables benefits such as massive parallelism and optimizations, which are difficult to exploit using traditional methods.

- Provide a relational interpretation of a constrained configuration system and prove the correctness of the satisfiabilitybased approach. In this process, the problem is also shown to be NP-complete, which justifies SAT as the canonical solution.

- Implement a prototype configurator based on the SAT method in a real industrial setting and compare the result to a few existing implementations using traditional approaches.

We begin by introducing the specification of an online configurator workflow.

\section{AN ONLINE CONFIGURATOR WORKFLOW}

All configurators are interactive if the system has non-empty constraints: the user inputs the desired configuration, and the configurator responds with a validation message indicating whether the configuration is admissible. Trivial systems with empty constraints are of little interest. Hence, in the rest of the paper, we will omit the description "interactive." A configurator is online when its user experience is reactive: it provides rich feedback on user input.

\subsection{User Workflow}

An online configurator user workflow is the following. 


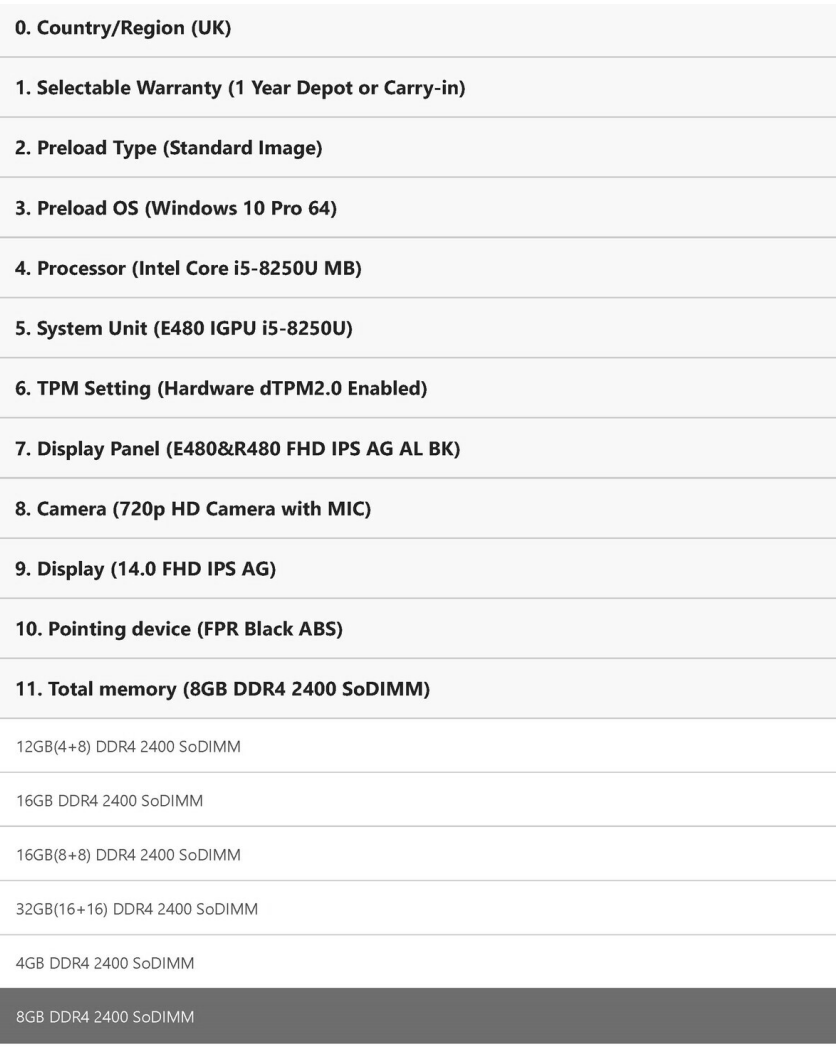

\section{GB(4+4) DDR4 2400 SoDIMM}

\section{Hard drive (Dummy HDD)}

13. Hard drive 2 (256GB SSD M.2 2280 NVMe OPAL2)

14. WiFi wireless LAN adapters (Intel 3165AC+BT 1x1 No vPro)

15. Battery (3cell $45 \mathrm{Wh})$

16. AC Adapter and Power Cord (65W USB-C 3Pin UK)
17. Keyboard Language
18. Publication Language
20. Preload Language
21. Adobe Elements optional (NONE)
22. Adobe Acrobat optional (NONE)

23. Microsoft Office (NONE)

Figure 1: An interactive, online configurator GUI example

(1) A graphical user interface (GUI) is initialized to display the list of all components and choices (e.g. Fig. 1).

(2) A default, bare-bones configuration is loaded. We assume the default configuration is always valid. Choices incompatible with the default configuration are indicated as unavailable on the GUI and cannot be chosen by the user.

(3) The user observes the displayed information on the GUI and proceeds to pick any available choice under a component. This counts as "a step." Additionally, the user can click on an unavailable choice to see a message that explains why this choice is unavailable.

(4) The configurator updates the current configuration to subsume the newly picked choice and updates the set of unavailable choices according to the change. The GUI is refreshed accordingly.

(5) The configuration is complete when all components are picked. Otherwise, go to step (3) and repeat.

By design, the configurator should never allow the user to enter the space of invalid configurations assuming the default configuration is valid. The feedback provided to the user by the configurator is twofold. First, the configurator must update the GUI to indicate all unavailable choices given the current configuration. This information needs to be up-to-date and accurate. Secondly, a user can examine why a specific choice is unavailable. A list of choices in the current configuration conflicting with the intended choice is provided.

\subsection{Structural Rules}

In addition to the user workflow above, a few structural rules are applied internally by the configurator. These rules are often needed to improve user experience and comply with business standards. They are ultimately arbitrary but are very common in industrial scenarios.

2.2.1 Mutual exclusivity $(M X)$ rule. Every component is only allowed to have one choice. In other words, all the choices under a single component are mutually exclusive. This rule makes sense because, for example, the user cannot pick two different CPUs for a single laptop configuration.

In cases where multiple choices are desired for the same component, the component is partitioned into multiple independent components beforehand. For example, suppose that a PC configurator allows up to three hard disk drives (HDD). Instead of having one multi-choice HDD component, three independent single-choice components HDD1, HDD2, HDD3 are offered instead.

2.2.2 Required (RQ) rule. All components must have a choice for the configuration to be complete. This rule is usually handled trivially in traditional designs but needs to be explicitly encoded in a satisfiability setting. Any constraint system is trivially satisfied if the configuration is empty.

In many real business settings, not all components are conceptually required, and the user is free to leave some components empty. For example, in a PC configurator, components such as mouse and keyboard are considered "add-ons." These components are not indispensable to the manufacturing process of a PC. The user is free not to purchase them. However, we notice that such behavior is only aesthetic. We do not lose expressivity in an all-requiring system. To emulate a partially-requiring system, we only need to insert beforehand a hidden choice (e.g., NONE) to all conceptually non-required components. All related constraint sets need to be amended as well. More details on this will be discussed later. 


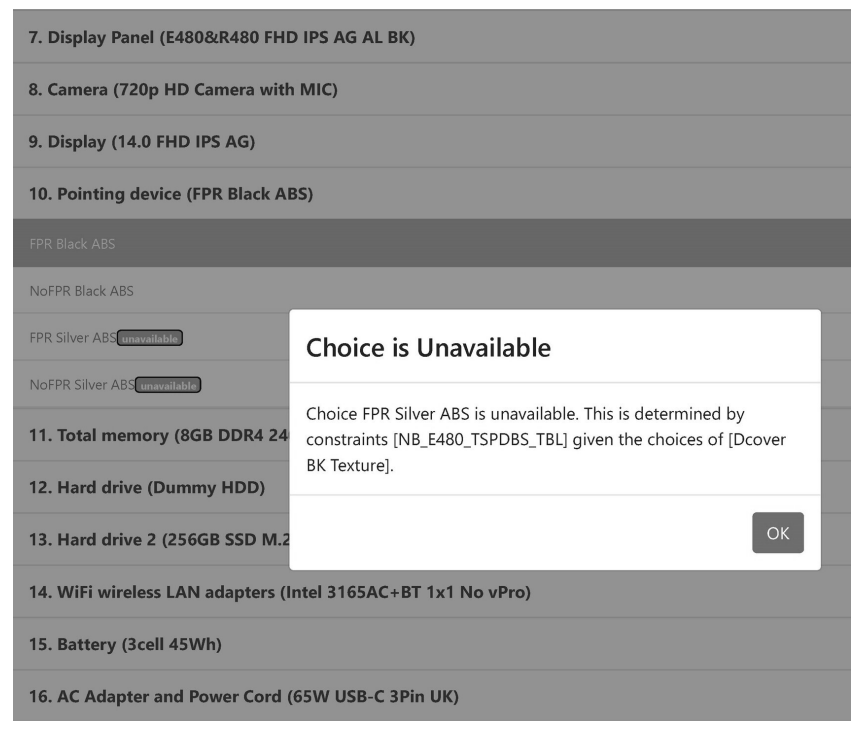

Figure 2: Example of a QUERY result interface

2.2.3 Automatic (AC) rule. Given that we have the $\mathrm{RQ}$ rule, it is only natural to expect the configurator to automatically pick the only remaining choice for the user when all other choices under the same component are unavailable. This rule is tricky since the automatically picked choice needs to be appended to the current configuration, which triggers a new round of configuration algorithm and GUI refresh. A fixpoint iteration is needed to stabilize the system whenever the AC rule is applied.

\subsection{Traditional Algorithm}

Given the above specification, a high-level, abstract algorithm is presented in Algorithm 1. It consists of two independent application programming interfaces (API). The UPDATE procedure updates internal states and provides feedback according to the latest configuration. The QUERY procedure searches for the reason an intended choice is unavailable.

The implementations of procedures such as Solve-Unavailable depend on the specific core constraint solving method used. The details are omitted here since there are many possible concrete implementations (e.g., constraint networks, logic programming). Similarly, procedures such as FIND-CONFLICT are also abstract and implementation-dependent. The procedure Synthesize-REAson formulates a user-friendly message from the conflicting choices.

\section{A SATISFIABILITY APPROACH}

The main procedure UPDATE is time-consuming due to the iterative fixpoint computation implementing the AC rule. It is unclear how this loop can be optimized in traditional methods such as constraint networks or pattern matching. These methods usually start from the total space of configurations and gradually prune the space guided by the constraints. To optimize away the loop, the core algorithm needs to detect the trigger condition of the AC rule midprocess and update the searching process in-place to reflect a new goal.

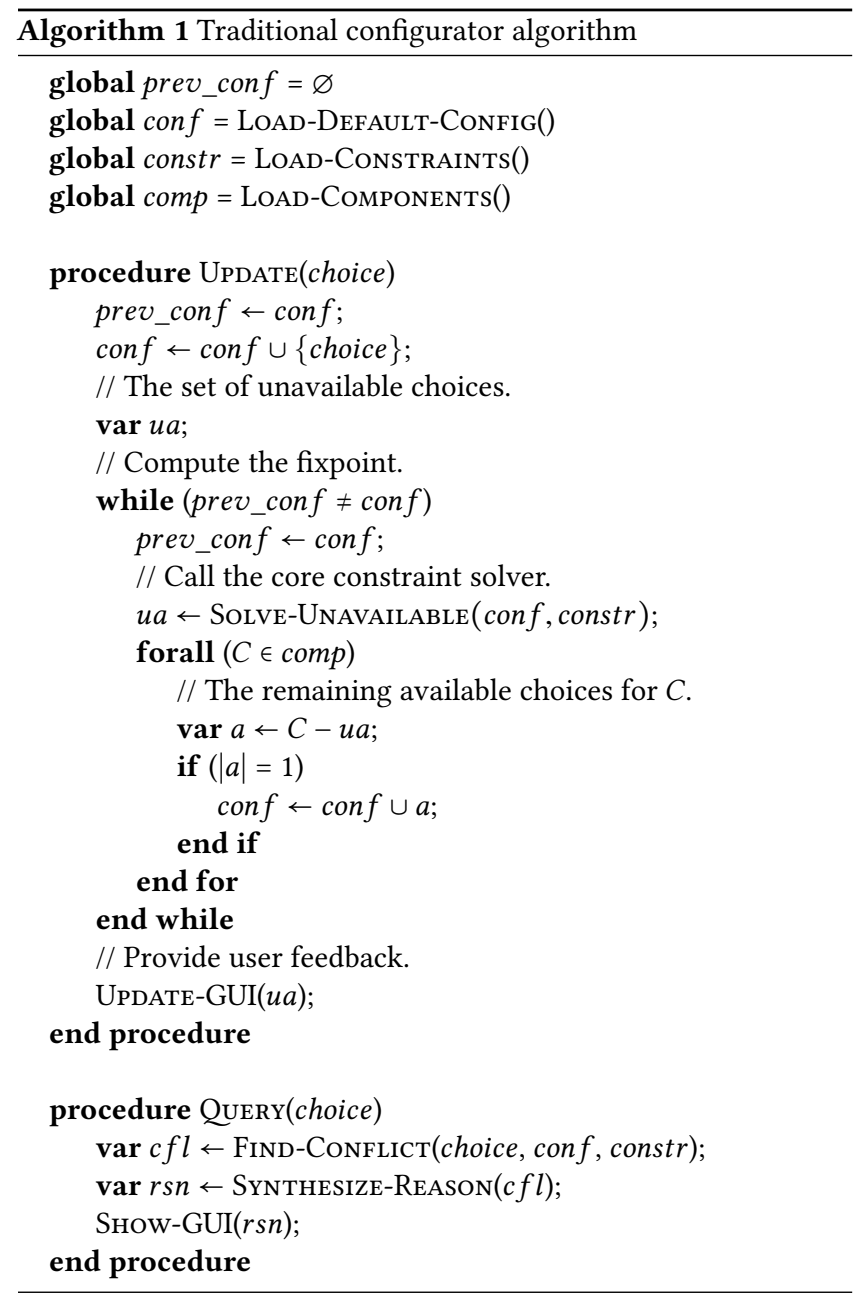

\subsection{Optimizing Procedural Logic by Deep Encoding}

If the problem is encoded using boolean logic, the loop can be completely optimized away by encoding the AC rule as an additional set of formulas. For each component $C_{i}$, we synthesize a new clause $A C_{i}$ where

$$
A C_{i}=\bigwedge\left\{\bigwedge_{c^{\prime} \in C_{i}-\{c\}} \neg c^{\prime} \Rightarrow c \mid c \in C_{i}\right\}
$$

The clause $A C_{i}$ informs the SAT solver that the unavailability of all other choices in the component $C_{i}$ implies picking the current choice. The newly introduced $A C$ clause does not increase the formula's overall complexity (no new variables are added). The fixpoint iteration is completely optimized away by this logical encoding.

\subsection{Optimizing by Massive Parallelization}

One potential drawback of the satisfiability approach is that the core solving procedure is "atomized." Traditional solvers based on constraint satisfaction or pattern matching can return the set of all unavailable choices after a single running session. This makes sense since these methods begin from the total space and reduce 


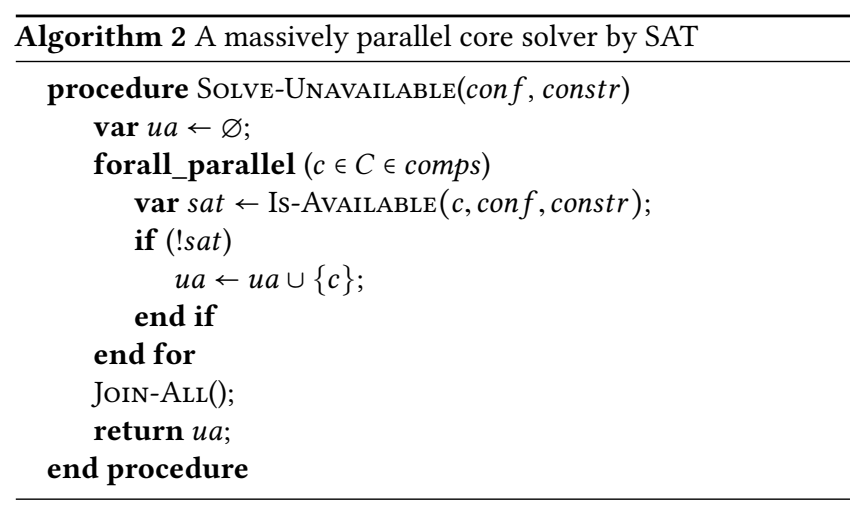

the space of choices by pruning heuristics. A SAT solver, on the other hand, returns a piece of binary information-whether the given formula is satisfiable or not. Therefore, the unavailability of all the component choices is solved individually.

On the other hand, however, we notice that each SAT solving procedure is independent and thus "embarrassingly parallelizable." This observation leads to a massively parallelized Algorithm 2. In the code, FORALL_PARALLEL distributes work to independent solvers running on different threads or computing nodes. The procedure IsAvAILABLE primarily consists of SAT formula creation and calls to off-the-shelf SAT solvers. Our experiments show that state-of-theart solvers such as Microsoft Z3[2] achieve impressive performance in this regard. More details will be presented in the evaluation section.

\subsection{Optimizing by Asynchronous Update}

We can further improve the system's performance by introducing small changes to the user workflow. In Algorithm 2, we notice that JoIN-AlL must wait for the slowest solving task to return even most tasks can finish quickly. We can hide the delay of the slowest task by making the GUI update asynchronous instead of monolithic. An asynchronous update means that as soon as any solver task returns, it reports its result (i.e., whether some choice $c$ is available) to the main program immediately. Then, the GUI is updated as soon as possible, even in a partial way.

The granularity of asynchronous update is the best if tasks are joined at the component level. This synchronization granularity leads to a more integral feedback experience: if a component is ready, the user knows all the information under the component is complete. Components still waiting to return are indicated on the GUI using unique visual markers to inform the user that the desired information is temporarily unavailable.

There is another important motivation for component-level synchronization. Namely, the main program must check the precondition of the AC rule when a component is ready and then add the new choice to the configuration if the AC rule is admissible. The reason is that the AC rule is only encoded implicitly in the formula but not reflected in the configurator program's state. The main program's state needs to be kept consistent with the solver's explicit and implicit results.

In an asynchronous system, the user no longer has to wait for the slowest solver task to finish at each step. Although the slowest task must return before the configurator begins the next iteration, the user experience is now pipelined, and the overall efficiency is increased.

\section{RELATIONAL INTERPRETATION OF CONSTRAINTS}

This section provides a theoretical formulation of the configurator problem, which justifies SAT solving as a solution. We first formalize the configurator problem using a relational model. Then we establish a correspondence between the relational model and SAT solving.

\subsection{Constraint}

A constraint $R$ is a set of relations defined on some set of components.

$$
R^{k}: \prod_{1 \leq i \leq k} C_{i}
$$

where $k$ is the arity of the constraint, and $C_{i}$ denotes the set of all possible choices for the component $C_{i}$. As an example,

$$
\begin{aligned}
& R^{2}: \text { CPU } \times \text { Memory } \\
& =\{(32 \mathrm{bit}, 4 \mathrm{G}),(64 \mathrm{bit}, 4 \mathrm{G}),(64 \mathrm{bit}, 8 \mathrm{G}),(64 \mathrm{bit}, 16 \mathrm{G})\}
\end{aligned}
$$

The system consists of arbitrarily many constraints. Each constraint has an arbitrary arity. The domain of a constraint $D(R)$ is the set of component names in this constraint. Taking the above as an example, $D\left(R^{2}\right)=\{$ CPU, Memory $\}$.

\subsection{Join and Union Operators}

We define two binary relational operators on constraints-the join operator $\otimes$ and the union operator $\oplus$.

4.2.1 Join. Given constraints $R_{1}, R_{2}$, the join operator combines both constraints, and the resulting constraint set is the Cartesian product of the two sets of relations. If $D\left(R_{1}\right)$ and $D\left(R_{2}\right)$ overlap, only compatible relations are preserved in the result. For example, by joining $R_{1}: \mathrm{CPU} \times$ Memory and $R_{2}:$ Memory $\times$ Motherboard, the relation (32bit, $4 \mathrm{G}) \in R_{1}$ is incompatible with $(8 \mathrm{G}, \mathrm{M} 1) \in R_{2}$. As another example, (32bit, $4 \mathrm{G})$ is compatible with (4G, M2) and yields (32bit, $4 \mathrm{G}, \mathrm{M} 2$ ) in the result $R_{1} \otimes R_{2}$. In fact, this operation is very similar to a database join. The expression $\otimes R_{i}$ refers to the join of all sets of constraints in the system.

4.2.2 Union. The union operator $\oplus$ is simply the set union of the two relation sets: $R_{1} \oplus R_{2}=R_{1} \cup R_{2}$. In case $D\left(R_{1}\right) \neq D\left(R_{2}\right)$, each relation is first extended with a wildcard notation to match the missing domains. The wildcard denotes the set of all possible choices in the corresponding component; it is a shorthand for writing out each combination explicitly. For example, $\{(4 \mathrm{G}, \mathrm{M} 2)\} \oplus\{(32$ bit, $4 \mathrm{G})\}=$ $\{(32$ bit $, 4 \mathrm{G}, *),(*, 4 \mathrm{G}, \mathrm{M} 2)\}: \mathrm{CPU} \times$ Memory $\times$ Motherboard. One can see that the union operator allows us to formulate an individual constraint set as $R=\left\{r_{1}, \ldots, r_{n}\right\}=\left\{r_{1}\right\} \oplus \ldots \oplus\left\{r_{n}\right\}$, where $r_{i}$ is each individual relation in the constraint set.

For both operators, we have $D\left(R_{1} \circ R_{2}\right)=D\left(R_{1}\right) \cup D\left(R_{2}\right)$ where $\circ$ is either $\otimes$ or $\oplus$. Using these two operators, we define a constraint system $\mathcal{R}$ as an expression consisting of individual relations $r$ or larger expressions connected using the two operators. 


\subsection{Configuration Validity}

A configuration is a set of arbitrary choices. We can interpret a configuration as a series of unary constraints. For example, a user configuration $\{64 \mathrm{bit}, 8 \mathrm{G}, \mathrm{M} 1\}$ can be interpreted as three singleton constraints $\{(64 \mathrm{bit})\},\{(8 \mathrm{G})\}$, and $\{(\mathrm{M} 1)\}$. Denote the join of all configuration-induced constraint as $\otimes G_{i}$.

For our purpose, the system $\mathcal{R}$ is defined as the join of all a priori constraint sets $\otimes R_{i}$ and all configuration-induced constraints $\otimes G_{i}$.

$$
\mathcal{R}=\bigotimes R_{i} \otimes \bigotimes G_{i}
$$

$\mathcal{R}$ can be seen as either an expression or a constraint set as the value of the expression. We say a configuration is valid if $|\mathcal{R}|>0$. It is invalid otherwise.

\section{CORRESPONDENCE WITH CLASSIC CSP}

The relational interpretation coincides with the classic notion of constraint satisfaction (CSP)[8] in the sense that

\section{$\mathcal{R}$ is equivalent to the set of all valid configurations.}

Proof. To show this, we use induction on the evaluation of an expression connected by the join operator $\otimes R_{i}$. Denote the domain of components of a constraint as $D(R)$. The base case scenario is a single constraint set $R_{0}$. By the definition of constraint, a configuration with regard to components $D\left(R_{0}\right)$ is valid if and only if it is in the set of $R_{0}$. Hence, the base case holds.

Inductively, suppose $\bigotimes_{i<j} R_{i}$ is equivalent to the set of all valid configurations wrt. $D\left(\otimes_{i<j} R_{i}\right)$. Need to show that $\left(\otimes_{i<j} R_{i}\right) \otimes R_{j}$ is equivalent to the set of all valid configurations wrt. $D\left(\otimes_{i \leq j} R_{i}\right)$. This is true because of the semantics of join: Cartesian products are formed between the two sets of relations $\bigotimes_{i<j} R_{i}$ and $R_{j}$ so that all possible combinations are included. Compatibility is guaranteed by the operational definition of join. Hence, $\otimes_{i \leq j} R_{i}$ is equivalent to the set of all valid configurations wrt. the domain $D\left(\otimes_{i<j} R_{i}\right) \cup$ $D\left(R_{j}\right)=D\left(\otimes_{i \leq j} R_{i}\right)$.

The only remaining bit is to show that this applies to the total domain (i.e. the set of all possible component names): it is possible that some components do not appear in $\otimes R_{i}$. We can obtain a version of $D\left(\otimes R_{i}\right)$ with regard to the total domain by unioning with an empty constraint set where the empty set is defined on the total domain. The result is $\otimes R_{i}$ amended by a series of wildcard notations on all the missing domains. We check that this is consistent with all valid configurations: missing domains are unconstrained and can be chosen freely.

\section{CORRESPONDENCE WITH SATISFIABILITY}

A boolean formula consists of boolean literals (a boolean variable or its negation) or larger expressions joined by the logical operators $\wedge$ and $\vee$. The goal of a boolean satisfiability problem (i.e. SAT) is to decide whether there exists a boolean assignment to all the variables in the formula (aka. a solution) such that the formula evaluates to true. The problem of SAT is known to be NP-complete. It is unlikely that a general, efficient algorithm would ever be discovered. Nevertheless, decades of research have gone into finding SAT solving heuristics, and state-of-the-art solvers can perform very efficiently on a large variety of inputs [4].
This section shows a correspondence between the relational interpretation presented in the previous section and boolean satisfiability solving, which formally justifies using SAT as a solution to the configurator problem. First, we define a translation function [[.] that maps a join/union expression to a boolean formula. Second, we show that the solution set of the resulting boolean formula preserves the exact relations of the original constraint set.

\subsection{Mapping Constraints to Formulas}

Define function [[.]]. This function maps $\otimes$ to $\wedge$, and it maps $\oplus$ to $\vee$. Individual relations are mapped to $\wedge$-clauses connecting its pure literals. More specifically,

(1) An individual relation in $R$ is mapped to a clause connected by $\wedge$. That is, $\left[\left[\left(c_{1}, \ldots, c_{k}\right)\right]\right]=c_{1} \wedge \ldots \wedge c_{k}$. In the same process, choices $c_{i}$ become boolean variables with the same name.

(2) A constraint set $R=\left\{r_{1}, \ldots, r_{n}\right\}$ is mapped to a clause formed by combining all its individual item clauses using the logical OR $\vee$. That is, $[[R]]=\left[\left[r_{1}\right]\right] \vee \ldots \vee\left[\left[r_{n}\right]\right]$.

(3) The system of all constraints are mapped to a formula that connects the clauses of each constraint $R$ with the logical AND $\wedge$. That is, $\left[\left[\otimes R_{i}\right]\right]=\wedge\left[\left[R_{i}\right]\right]$.

\subsection{Model Correspondence}

We now show that the solutions of the translated formula, in conjunction with the MX rule, represent the exact set of relations in the original constraint system. Assuming a constraint system $\mathcal{R}$ and boolean formula $S=[[\mathcal{R}]]$, we claim

\section{The solutions to formula $S \wedge M X$ is the set of $\mathcal{R}$.}

$M X$ refers to the mutual exclusivity rule encoded as a SAT formula. The detail of this formula is specified in the next section.

Proof. To see this, we use induction on the definition of the function [[.]. The base case is an individual relation $\left(c_{1}, \ldots, c_{k}\right)$ in some $R$ such that $S=\left[\left[\left(c_{1}, \ldots, c_{k}\right)\right]\right] \wedge M X=c_{1} \wedge \ldots \wedge c_{k} \wedge M X$. The only solution to $c_{1} \wedge \ldots \wedge c_{k}$ is when all the literals are true, which is exactly $\left\{\left(c_{1}, \ldots, c_{k}\right)\right\}$. The solution $\left(c_{1}, \ldots, c_{k}\right)$ satisfies $M X$ because the domain set is distinct for a relation: no more than one choice/variable under the same component can appear in the same relation. Inductively, there are two cases. We argue both cases hold by demonstrating that the inductive formula construction simulates the operational definition of the join/union operators respectively.

a) Need to show that the solutions to $S=([[\mathcal{A}] \wedge \llbracket \mathcal{B} \rrbracket) \wedge M X$ is $\mathcal{R}=\mathcal{A} \otimes \mathcal{B}$. By induction hypothesis $(\mathrm{IH})$, the solutions to $[[\mathcal{A}]$ (resp. $[[\mathcal{B}])$ is exactly $\mathcal{A}(\operatorname{resp} . \mathcal{B})$. The solution to $[[\mathcal{A}]] \wedge[[\mathcal{B}]]$ is the concatenation of Cartesian product of the solutions from both $[[\mathcal{A}]]$ and $[[\mathcal{B}]]$. This simulates the operational definition of the $\otimes$ operator. What remains is to show that only compatible relations are preserved. This is guaranteed by the $\wedge M X$ clause: the solutions to $[[\mathcal{A}]] \wedge[[\mathcal{B}]] \wedge M X$ exclude those concatenations in $[[\mathcal{A}] \wedge \llbracket[\mathcal{B} \rrbracket$ where two different choice variables under the same component are present. In other words, either $D(\mathcal{A})$ and $D(\mathcal{B})$ are completely disjoint, or the choice variables must be exactly the same in the concatenated solution under the same component. This is identified with the operational definition of the $\otimes$ operator. 
b) Need to show that the solutions to $S=([[\mathcal{A}] \vee \llbracket[\mathcal{B}]) \wedge M X$ is $\mathcal{R}=\mathcal{A} \oplus \mathcal{B}$. By IH, the solutions to $[[\mathcal{A}]]$ (resp. [[B] $]$ ) is exactly $\mathcal{A}$ (resp. $\mathcal{B}$ ). First, the domains of the solutions of each set are extended to match up with $D(\mathcal{A}) \cup D(\mathcal{B})$. This extension simulates the "wildcard" extension (section 4.2.2) in the case $D(\mathcal{A})$ and $D(\mathcal{B})$ are different, which is again guaranteed by the $\wedge M X$ clause: the choice variables in all the extended domains can vary freely but only on a per-component basis. Additionally, it should be clear that the solution to $[[\mathcal{A}]] \vee[[\mathcal{B}]$ is the set union of the extended solutions from both $[[\mathcal{A}]]$ and $[\mathcal{B}]$. Therefore, this construction is identified with the operational definition of the $\oplus$ operator, and the conclusion follows.

\subsection{Correctness and Canonicality}

As an easy corollary of the above propositions, we have that

$$
\text { Given } S=[[\mathcal{R}]], S \wedge M X \text { is satisfiable iff. }|\mathcal{R}|>0
$$

This formally justifies the use of satisfiability solving as a configurator algorithm.

As another consequence, we confirm that the configurator problem is NP-complete: we already showed that the problem could be transformed into an equivalent SAT problem; as the other direction, to transform a SAT formula into a configurator problem, simply take the conjunctive normal form (CNF) form of the formula and use the truth tables of all the clauses as constraint sets. This converse translation procedure is rather straightforward and well-known. Therefore, the configurator problem admits no efficient solution in general unless $\mathrm{P}=\mathrm{NP}$. It shows that SAT solving is not only a viable, natural solution, but it is in fact the canonical solution.

\section{IMPLEMENTATION}

We now go over the concrete implementation of the procedure IsAvailable used in Algorithm 2. This is summarized in Algorithm 3. This procedure first constructs formulas according to the given constraints and user configuration. Then, it feeds the final combined formula to an off-the-shelf SAT solver such as Z3.

\subsection{Construction of Boolean Formula}

The previous section elaborated the theory behind a boolean satisfiability formulation of the configurator problem. The exact construction of the SAT formula should be straightforward. Nevertheless, we cover them in this subsection for the sake of completeness.

The construction of the boolean formula has two parts. The first part concerns the constraint system itself plus the current user configuration. According to the previous section, the formula of the first part is $[[\mathcal{R}]]$. The second part has to do with the implementation of the three structural rules. The total formula refers to the final formula fed to the SAT solver. In practice, most parts of the formula can be cached to avoid redundant construction across configurator iterations.

7.1.1 Encoding the $M X$ rule. As a reminder, we need the mutual exclusivity rule to enforce a single-choice mechanism. This is done by using a set of formulas $M X_{i}$ for every component $C_{i}$ where

$$
M X_{i}=\bigwedge\left\{c \Rightarrow \bigwedge_{c^{\prime} \in C_{i}-\{c\}} \neg c^{\prime} \mid c \in C_{i}\right\}
$$

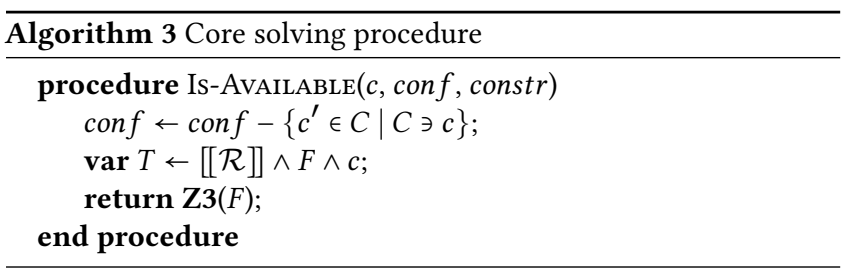

7.1.2 Encoding the $R Q$ rule. The required (RQ) rule is needed to eliminate partial configurations. A partial configuration does not contain a choice for at least one component. We can forbid partial configurations by adding the following formula for each component $C_{i}$.

$$
R Q_{i}=\bigvee_{c \in C_{i}} c
$$

7.1.3 Encoding the AC rule. As discussed in sections 2 and 3, the automatic (AC) rule selects the only remaining available choice of any component. This serves as a complement to the RQ rule and is implemented by the formula

$$
A C_{i}=\bigwedge\left\{\bigwedge_{c^{\prime} \in C_{i}-\{c\}} \neg c^{\prime} \Rightarrow c \mid c \in C_{i}\right\}
$$

The formula of all the structural rules is the following, which we denote as $F$.

$$
F=\bigwedge_{i}\left(M X_{i} \wedge R Q_{i} \wedge A C_{i}\right)
$$

The core solving mechanism Is-AvAILABLE is implemented by supplying a total formula $T$ to a SAT solver. $T$ is assembled using both $[[\mathcal{R}]$ and the structural rule formula $F . c$ is the choice under examination and thus appended at the end. Importantly, we must subtract all the choices under the same component as $c$ beforehand. This is to avoid a direct violation of the $M X$ rule. Since $c$ is the "intended" choice, discounting the current choice under the same component (if exists) is interpreted as deselecting the current choice and then picking $c$. This makes sense in the configurator workflow. If $T$ is satisfiable, it proves that the choice $c$ is available as part of a valid configuration. $c$ is unavailable otherwise.

\subsection{Emulating Non-required Components}

Many real-world configurators allow partial configurations for addon components. Due to the RQ rule, our system only supports complete configurations. We can emulate partial configurations by the following preprocessing. For every conceptually non-required component, we insert an artificial choice NONE into the component's choice set. Additionally, modify each constraint set such that for any $R: \prod C_{i}$ and any non-required $C_{i}$, we append an extra relation with NONE in the corresponding component for all relations. For example, suppose $\left(c_{1}, c_{2}\right) \in R: C_{1} \times C_{2}$, and $C_{1}$ is non-required. Append an extra relation (NONE, $c_{2}$ ) to the constraint set. The appended constraints ensure that NONE is always part of a valid configuration for non-required components. 
Table 1: Comparison with two other in-house implementations

\begin{tabular}{ccccc}
\hline Core method & Core solver performance / ms & External cache size / MB & Correctness & Core code size / LOC \\
\hline constraint-network & $150-1,500$ & $>5,000$ & unclear & $>10,000$ \\
pattern-matching & $25-250$ & $>400$ & unclear & $>5,000$ \\
SAT (Z3, four cores, 1,417 tasks) & $1,633-1,705$ & 0 & proven & 280 \\
\hline
\end{tabular}

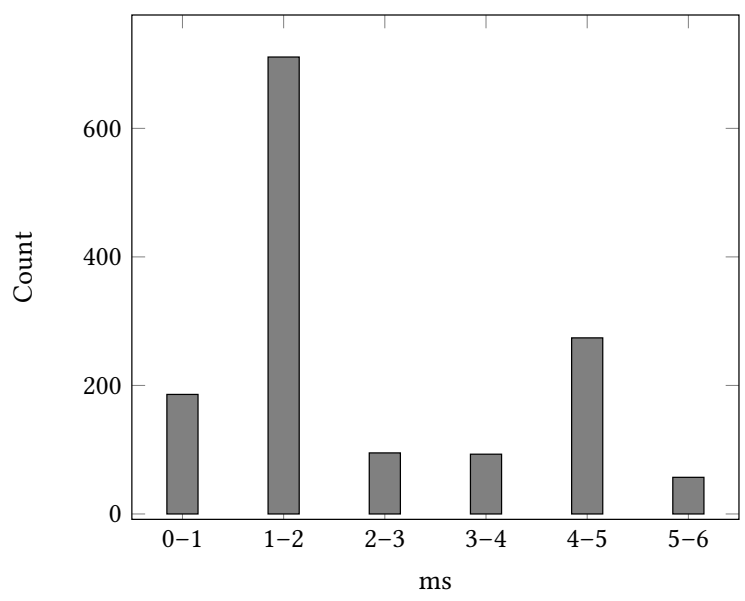

Figure 3: Individual solver task response time distribution

\subsection{Implementing the Query Interface}

An online configurator has two user interfaces: UPDATE and QUERY. It turns out that the function of QuERY is already embedded in many SAT solvers such as Z3 as a minimal unsatisfiable core (MUC) extraction procedure[5]. Hence, QUERY consists of a direct call to the Z3 MUC extraction interface. The solver returns a best-effort minimal unsatisfiable subformula (i.e. the core) of the total formula. The core contains the "reason" why a configuration is invalid: the minimality of the formula localizes the set of constraint clauses directly related to the unsatisfiability. The core is then filtered and analyzed by additional routines to synthesize a friendly message to the user. Refer to Fig. 2 for an example.

\section{EVALUATION}

We implemented the above configurator design during our stay at a global PC manufacturer.

\subsection{Background and Setup}

The client-facing, non-solver part of our prototype is coded in Java OpenJDK 1.8.0 version 252 and runs on an Intel i5-9600 at $3.70 \mathrm{GHz}$ The core solver choice is the Microsoft Z3 solver based on the 64-bit version 4.8.8[2][10]. The Java code is connected to native Z3 instances using Java Native Interface (JNI) with the help of a specialised library[6][13]. Due to the limited time and resources available to us, we only implemented a synchronous parallel configurator (not the asynchronous configurator in section 3.3).

The experiments were conducted on the configuration of one of the largest PC products in size with the code name "30BA." The total formula of 30BA contains 1,417 variables and 18,970 basic $\bigvee$-clauses with only literals. The number of clauses in conjunctive normal form (CNF) is estimated to be much larger.

We compare our prototype to two existing in-house implementations based on non-satisfiability methods concerning factors such as core solver performance, external cache use, correctness, and code size. The results are listed in Table 1 . The data of existing implementations are based on reported numbers in the documentation. "Core solver performance" refers to the solver response time of an entire configurator iteration. Additionally, we collected the response time of all individual solver tasks using the SAT method in a sample running session based on the SAT method and summarized the data in the Fig. 3 histogram. The $\mathrm{x}$-axis is the response time for a single task. The y-axis is the number of tasks that fall into all the $1 \mathrm{~ms}$ response time ranges. One can see that the majority of tasks finish under $2 \mathrm{~ms}$. The average solving time is approximately $2 \mathrm{~ms}$. This data indicate how Z3 performs per each SAT formula.

\subsection{Discussion}

We observe that despite decent individual task performance, the SAT implementation does not achieve a faster overall performance than existing methods. This is because our prototype currently does not scale well with regard to multi-core processing: in the prototype, each core has to instantiate a separate Z3 instance via JNI, which introduces a significant overhead and prevents further scaling. On our six-core machine, experiments show that parallel speedup ceases at four cores. We expect a fine-tuned, productionready implementation to scale much better since each solver task is completely independent.

Engineering-wise, the ultimate goal is a non-blocking, asynchronous server. The key challenge is to minimize the overhead of interfacing Z3 instances. Ideally, the Z3 instances should be "hot": always in a running state waiting for input, instead of being shut down after each iteration and restarted in the next, a high cost on solving time. Additionally, the server architecture should be asynchronous. Upon completion of an individual task, the result of the task is immediately returned to the client-facing GUI, as opposed to waiting for all tasks to finish. In this situation, the average feedback time for the client should be similar to the average core solving time of the SAT method, which is approximately $2 \mathrm{~ms}$ according to the data we collected in Fig. 3. This is a speedup compared to existing implementations with a best-case response time of $25 \mathrm{~ms}$ (Table 1).

Our method is provably correct, which is an important and unique advantage. The SAT-based method also does not require any additional external cache space. Other methods tend to require considerable extra memory. Finally, the SAT method is much easier to implement and maintain. The core implementation in our design only takes up 280 lines of code. This should not be surprising since our method is declarative while the others are procedural. 


\section{FUTURE WORKS}

Important future works include upgrading and fine-tuning our prototype implementation to be production-ready and ensure the server scales well.

Many SAT solvers like Z3 have an optional mode where the solver returns a sample boolean assignment that satisfies the formula (if the formula is satisfiable). This technique may be used to prune the space of solver work.

We are also interested in the reason Z3 performs well in our problem domain. In our experiments, several other off-the-shelf solvers besides Z3 were tried. However, none comes close to Z3 in performance. It remains as future work to analyze Z3 internal and explain its fitting characteristics to our problem domain.

\section{RELATED WORKS}

A configurator problem is traditionally formalized as a CSP problem. In CSP, variables correspond to components, and variable domains correspond to choices. Usually, $k$-ary $(k>2)$ constraints are first converted to binary constraints with binarization techniques[1] and solved using constraint network heuristics[8]. However, binarization introduces new variables into the system to emulate non-binary constraints. This increases the complexity of the SAT formula. The number of new variables is at least proportional to the size of the constraints in the system. It is not clear whether binarization-based algorithms scale well when the number of constraints is large, and the constraint sets are dense (often seen in real business scenarios). Plus, it is often more desirable to preserve the structure of the original constraints for debugging and usability reasons.

Satisfiability-based methods were proposed in the past[7][11][14]. As demonstrated in the papers, SAT solving proves to be efficient for the core configurator problem, where the goal is to decide if the business constraints are satisfied given a user configuration. For a configurator that requires an online experience with rich feedback to the user at every step, more care and design need to be considered, which we presented in this paper.

Other methods exist, such as those based on pattern matching[3] and logic programming[12].

\section{CONCLUSION}

In this paper, we present a satisfiability approach to the online configurator problem. An online configurator consists of a GUI facility and main program that manages constraint solving. The user can look at all the components of a product on the GUI and make choices interactively in a step-wise manner. The main program ensures the user does not violate any a priori business constraints. Given a current working configuration, it refreshes the GUI to display the available choices that remain to the user. We formulate this problem as a SAT-solving procedure. Using satisfiability encoding, much procedural logic is also optimized away. The correctness of our approach is formally proven. Our approach is also superior in memory use and simplicity to traditional methods based on constraint networks and pattern matching. In order to achieve optimal performance, a proper distributed infrastructure is necessary. With such infrastructure, it is expected that our method scales well since each solver task is completely independent. Each task can also update the GUI asynchronously to further enhance speed and user experience.

\section{REFERENCES}

[1] Fahiem Bacchus and Peter Van Beek. 1998. On the conversion between nonbinary and binary constraint satisfaction problems. In AAAI/IAAI. 310-318.

[2] Leonardo De Moura and Nikolaj Bjørner. 2008. Z3: An efficient SMT solver. In International conference on Tools and Algorithms for the Construction and Analysis of Systems. Springer, 337-340.

[3] Charles L Forgy. 1989. Rete: A fast algorithm for the many pattern/many object pattern match problem. In Readings in Artificial Intelligence and Databases. Elsevier, 547-559.

[4] Jun Gu, Paul W Purdom, John Franco, and Benjamin W Wah. 1996. Algorithms for the satisfiability (SAT) problem: A survey. Technical Report. Cincinnati Univ oh Dept of Electrical and Computer Engineering.

[5] Ofer Guthmann, Ofer Strichman, and Anna Trostanetski. 2016. Minimal unsatisfiable core extraction for SMT. In 2016 Formal Methods in Computer-Aided Design (FMCAD). IEEE, 57-64.

[6] Egor George Karpenkov, Karlheinz Friedberger, and Dirk Beyer. 2016. JavaSMT: A unified interface for SMT solvers in Java. In Working Conference on Verified Software: Theories, Tools, and Experiments. Springer, 139-148.

[7] Wolfgang Küchlin and Carsten Sinz. 2000. Proving consistency assertions for automotive product data management. Fournal of Automated Reasoning 24, 1-2 (2000), 145-163.

[8] Vipin Kumar. 1992. Algorithms for constraint-satisfaction problems: A survey. AI magazine 13, 1 (1992), 32-32.

[9] John McDermott. 1982. R1: A rule-based configurer of computer systems. Artificial intelligence 19, 1 (1982), 39-88

[10] Microsoft. 2021. Z3Prover. https://github.com/Z3Prover/z3. Accessed: 2021-03-15.

[11] Carsten Sinz, Andreas Kaiser, and Wolfgang Küchlin. 2003. Formal methods for the validation of automotive product configuration data. Ai Edam 17, 1 (2003), 75-97.

[12] Timo Soininen and Ilkka Niemelä. 1999. Developing a declarative rule language for applications in product configuration. In International Symposium on Practical Aspects of Declarative Languages. Springer, 305-319.

[13] SoSy-Lab. 2021. JavaSMT. https://github.com/sosy-lab/java-smt. Accessed: 2021-03-15.

[14] Alexey Voronov. 2013. On formal methods for large-scale product configuration. Chalmers University of Technology. 